\title{
Gastrectomía laparoscópica casi total con preservación de la rama ascendente de la arteria gástrica izquierda y un $5 \%$ de remanente gástrico. ¿Existe relación con las complicaciones de la anastomosis?
}

\author{
Almost total laparoscopic gastrectomy with preservation of the ascending \\ branch of the left gastric artery and $5 \%$ gastric remnant. \\ Is there a relationship with the complications of the anastomosis?
}

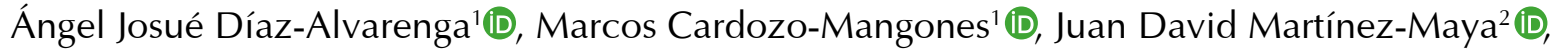
Juan Pablo Gómez-Vélez² $\mathbb{D}$, Santiago Gómez-Correa² $\mathbb{D}$, Mauricio Esteban Valencia-Agudelo² $\mathbb{D}$,

Felipe Solórzano-Ferrer ${ }^{2} \mathbb{D}$, José Hugo Arias-Botero ${ }^{3} \mathbb{D}$, Jorge Alberto Bernal-Mesa ${ }^{4} \mathbb{D}$

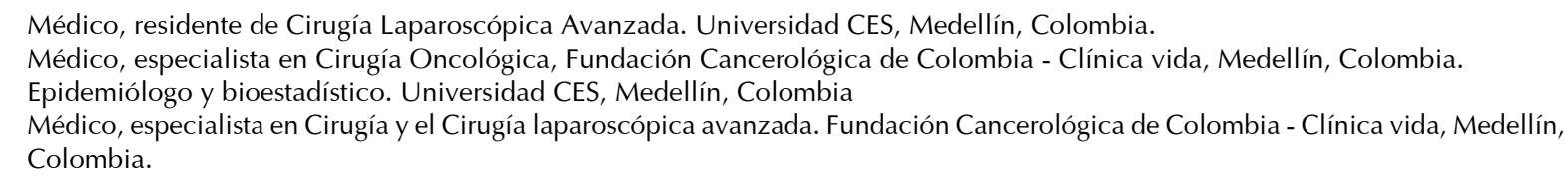

\section{Resumen}

Introducción. Al realizar una gastrectomía distal, el remanente gástrico es irrigado por los vasos gástricos cortos. En este estudio presentamos una serie de casos de gastrectomía distal laparoscópica, con la variante de preservar la rama superior de la arteria gástrica izquierda, con un remanente gástrico pequeño.

Métodos. Estudio observacional con intensión analítica, seguimiento de una cohorte retrospectiva de pacientes con cáncer gástrico sometidos a gastrectomía distal radical laparoscópica entre enero 2017 y junio 2019. Se incluyeron todos los pacientes con cáncer gástrico a quienes se les realizó gastrectomía distal radical por laparoscopia. Se estudiaron variables clínicas de los pacientes, variables intraoperatorias, información relacionada con el informe de patología y las complicaciones de la anastomosis gastro-yeyunal.

Resultados. Se revisó un total de 6I historias clínicas de pacientes sometidos a gastrectomía distal por laparoscopia, entre quienes se encontró la preservación de la rama ascendente de la arteria gástrica izquierda en el 73,8 \% de los pacientes $(n=45)$. El promedio de ganglios resecados fue de 24 . Se presentaron un total de io complicaciones, ocho relacionadas con la anastomosis (tres estenosis, cuatro filtraciones y un paciente con hemorragia del tracto

Fecha de recibido: 19/06/2020 - Fecha de aceptación: 21/08/2020

Correspondencia: Ángel Josué Díaz Alvarenga. Carrera. 30 \# 5 F - 185, Universidad CES, Medellín, Colombia. Teléfono: 3505711921 Correo electrónico: diaz.aj88@gmail.com

Citarcomo: Díaz-AlvarengaÁJ, Cardozo-MangonesM, Martínez-MayajD, Gómez-VélezJP, Gómez-CorreaS, Valencia-AgudeloME, etal. Gastrectomía laparoscópica casi total con preservación de la rama ascendente de la arteria gástrica izquierda y un $5 \%$ de remanente gástrico. ¿Existe relación con las complicaciones de la anastomosis? Rev Colomb Cir. 2020;35:583-92. https://doi.org/10.30944/20117582.499

Este es un artículo de acceso abierto bajo una Licencia Creative Commons - BY-NC-ND https://creativecommons.org/licenses/by-ncnd/4.0/deed.es 
digestivo superior), y dos con colecciones intrabdominales. En el análisis estadístico no se encontró relación entre la preservación de la rama ascendente de la arteria gástrica izquierda con la presencia de complicaciones.

Discusión. La variación que presentamos de la técnica de la gastrectomía distal por laparoscopia cumple con los requisitos oncológicos en los pacientes con cáncer gástrico y no se relaciona con complicaciones de la anastomosis gastró-yeyunal.

Palabras clave: neoplasias gástricas; adenocarcinoma; gastrectomía; laparoscopia; complicaciones posoperatorias.

\begin{abstract}
Introduction. When performing a distal gastrectomy, the gastric remnant is supplied by the short gastric vessels. In this study we present a series of cases of laparoscopic distal gastrectomy, with the variant of preserving the superior branch of the left gastric artery, with a small gastric remnant.

Methods. Observational study, follow-up of a retrospective cohort of patients with gastric cancer who underwent laparoscopic radical distal gastrectomy between January 2017 and June 20I9. All patients with gastric cancer who underwent laparoscopic radical distal gastrectomy were included. Clinical variables of the patients, intraoperative variables, information related to the pathology report and complications of the gastro-jejunal anastomosis were studied.
\end{abstract}

Results. A total of 6I medical records of patients undergoing laparoscopic distal gastrectomy were reviewed; preservation of the ascending branch of the left gastric artery was found in $73.8 \%$ of the patients $(n=45)$. The average number of lymph nodes resected was 24. There were a total of Io complications, eight related to the anastomosis (three strictures, four leaks and one patient with bleeding from the upper digestive tract), and two patients who presented intra-abdominal collections. No statistical relationship was found between the preservation of the ascending branch of the left gastric artery and the presence of complications.

Discusion. The variation that we present of the laparoscopic distal gastrectomy technique meets the oncological requirements in patients with gastric cancer and is not related to complications of the gastrojejunal anastomosis.

Keywords: stomach neoplasms; adenocarcinoma; gastrectomy; laparoscopy; postoperative complications.

\section{Introducción}

La resección gástrica y la linfadenectomía son actualmente la única terapia con intención curativa en los pacientes con cáncer gástrico ${ }^{\perp}$ La técnica quirúrgica clásica de gastrectomía distal, es aquella en la que la resección del estómago incluye el píloro mientras el cardias es preservado, la arteria gástrica izquierda es seccionada y el remanente gástrico es irrigado por los vasos gástricos cortos ${ }^{2}$.

La arteria gástrica izquierda se divide en una rama anterior y en una rama posterior; la rama anterior recorre la pared anterior del estómago hacia la curvatura mayor y la rama posterior recorre la curvatura menor y se une con la arteria gástrica derecha. Por su recorrido, estas ramas, también pueden ser llamadas, rama ascendente y rama descendente, respectivamente ${ }^{3}$.

Existen estudios en los que se informan mayores complicaciones técnicas, al momento de encontrar variantes anatómicas en las arterias peri-gástricas ${ }^{4}$, por lo cual, en algunas instituciones de otros países, realizan estudios de imágenes de alta resolución para identificar todas estas variantes anatómicas ${ }^{5}$. De acuerdo al resultado de estos estudios se sabe que el $90 \%$ de la población tiene la clásica irrigación previamente descrita.

Una de las complicaciones más temidas en una gastrectomía total, es la filtración de la anastomosis esófago-yeyunal. En la década de los 
ochenta, se modificó la técnica y se introdujo el concepto de gastrectomía casi total ${ }^{6,7}$, en la cual se deja un remanente gástrico del $5 \%$ y se realiza una anastomosis gastro-yeyunal, teóricamente mejor irrigada.

En nuestra institución, se realiza actualmente una variante de la técnica con gastrectomía casi total, dejando un reservorio gástrico del $5 \%$ con preservación de la rama ascendente de la arteria gástrica izquierda (RAAGI), combinando la radicalidad de una gastrectomía total con una probable mejor irrigación del remanente gástrico y una probable diminución de las complicaciones de la anastomosis esófago-yeyunal.

El objetivo del presente estudio fue describir una cohorte de pacientes llevados a gastrectomía casi total radical por laparoscopia, con preservación de la rama ascendente de la arteria gástrica izquierda como variación de la técnica, evaluando su asociación con las complicaciones de la anastomosis gastro-yeyunal.

\section{Métodos}

Se trata de un estudio observacional con intención analítica, para seguimiento de una cohorte retrospectiva de pacientes con cáncer gástrico sometidos a gastrectomía distal radical laparoscópica, entre enero de 2017 y junio de 20I9, en la Fundación Cancerológica de Colombia - Clínica Vida, de la ciudad de Medellín, Colombia. Esta institución es un centro de referencia de pacientes oncológicos, que cuenta con cirujanos entrenados en técnicas quirúrgicas de laparoscopia avanzada, y en ella se realizan procedimientos mínimamente invasivos en el área de la oncología digestiva, tratando patologías como cáncer de esófago, estómago, colon y recto.

Se excluyeron los pacientes que tuvieron conversión del procedimiento laparoscópico a cirugía abierta y aquellos con información insuficiente en el expediente clínico. Se estudiaron variables clínicas de los pacientes, variables intraoperatorias, información relacionada con el informe de patología y las complicaciones de la anastomosis gastro-yeyunal (estenosis, filtración, hemorragia y colecciones intraabdominales).

\section{Descripción de la técnica}

La técnica se realiza bajo anestesia general, se introduce una sonda de Foley de manera rutinaria, y se coloca al paciente en posición francesa, con el cirujano entre las piernas del paciente y un ayudante a cada lado. El neumoperitoneo se logra por técnica abierta. Se coloca un trocar umbilical y otro en el flanco izquierdo, ambos de I $2 \mathrm{~mm}$, y tres trocares de $5 \mathrm{~mm}$, uno subxifoideo que permite la retracción del hígado, otro en el flanco derecho y otro izquierdo, más lateral que el colocado inicialmente. Se realiza un lavado peritoneal con $250 \mathrm{ml}$ de solución salina, de los cuales se recuperan $50 \mathrm{ml}$ que son enviados a patología.

Omentectomía: Se inicia la disección liberando en su totalidad el epiplón mayor, desde el ángulo esplénico hasta el ángulo hepático del colon transverso, respetando durante todo el tiempo el mesocolon transverso, de esta manera se obtiene la linfadenectomía del grupo 4 .

La disección del epiplón mayor del lado derecho expone la cara anterior del páncreas, logrando así identificar y ligar los vasos gastroepiploicos derechos junto con el grupo linfático 6 , esto permite además exponer la parte posterior del píloro, creando así un túnel retroduodenal.

Sección duodenal y linfadenectomía de la arteria hepática: Se utiliza sutura mecánica para realizar la sección de la primera porción del duodeno, identificando y ligando la arteria gástrica derecha. Se realiza además la resección del grupo linfático 5 .

Se inicia la linfadenectomía de la arteria hepática distalmente, obteniendo los ganglios del grupo I2a y resecando en bloque los ganglios del grupo 8. La disección continua en sentido ascendente resecando en su totalidad, lo más cercano posible al hígado, el ligamento gastroduodenal y gastrohepático hasta identificar el pilar derecho, donde el grupo ganglionar I es resecado.

Linfadenectomía del tronco celiaco y de la arteria gástrica izquierda: Posteriormente se identifica el tronco celiaco y la arteria gástrica 
izquierda, se realiza el vaciamiento ganglionar de los grupos 7 y 9 respetando la RAAGI. En caso de encontrar dificultades técnicas para realizar un vaciamiento linfático adecuado que comprometa la radicalidad del procedimiento, por ejemplo, hemorragia o presencia de una enfermedad ganglionar importante a este nivel, no se preserva la RAGGI (figura I).

Disección del pilar izquierdo y sección de vasos gástricos cortos: la disección continua en el pilar izquierdo con la linfadenectomía del grupo 2. En caso de lograr respetar la RAAGI, se realiza la sección de todos los vasos cortos, de lo contrario se procede con la técnica clásica, dejando un remanente gástrico irrigado por los vasos gástricos cortos.

Resección Gástrica: la gastrectomía se realiza con sutura mecánica, inicialmente desde la curvatura menor a $3 \mathrm{~cm}$ por debajo de la unión gastroesofágica, posteriormente calibrado con una bujía de 38 french se realiza otro corte con sutura mecánica en sentido longitudinal a la bujía, dejando así un remanente gástrico pequeño del $5 \%$ (figura 2).

Reconstrucción gastrointestinal: La reconstrucción del tracto gastrointestinal se realiza mediante una "Y de Roux", con una longitud de asa alimentaria de $60 \mathrm{~cm}$ y una asa bilio-pancreá-

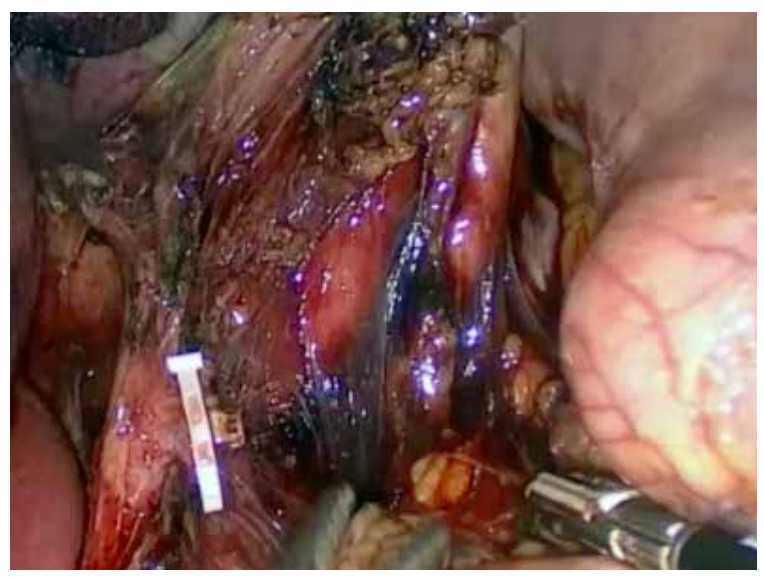

Figura 1. Resultado final de la linfadenectomía de la arteria gástrica izquierda con preservación de la rama ascendente y ligadura de la rama descendente. tica de $60 \mathrm{~cm}$. La anastomosis gastro-yeyunal se confecciona de una forma termino-lateral con sutura mecánica y se hace cierre manual de la enterotomía con sutura monofilamento no absorbible 2-o en dos planos. $\mathrm{O}$ bien, se realiza una anastomosis termino-lateral manual en dos planos posteriores con sutura no absorbible y uno anterior con sutura absorbible monofilamento 2-o. Rutinariamente se deja un dren cerca de la primera porción del duodeno. Se amplía la incisión umbilical, se coloca un dispositivo protector de herida y se extrae la pieza quirúrgica. Todos los puertos para trocares de más de Io $\mathrm{mm}$ son cerrados con puntos que incluyen la fascia aponeurótica.

\section{Análisis estadístico}

Las variables cualitativas son presentadas como frecuencias absolutas y relativas mediante tablas de frecuencia; las variables cuantitativas se presentan como medidas de tendencia central y medidas de dispersión (promedio con desviación estándar y mediana con rango intercuartílico, dependiendo de si se distribuye de forma normal o no).

Se comparan las características de los pacientes que presentaron complicaciones de la anastomosis gastro-yeyunal y los que no, apli-

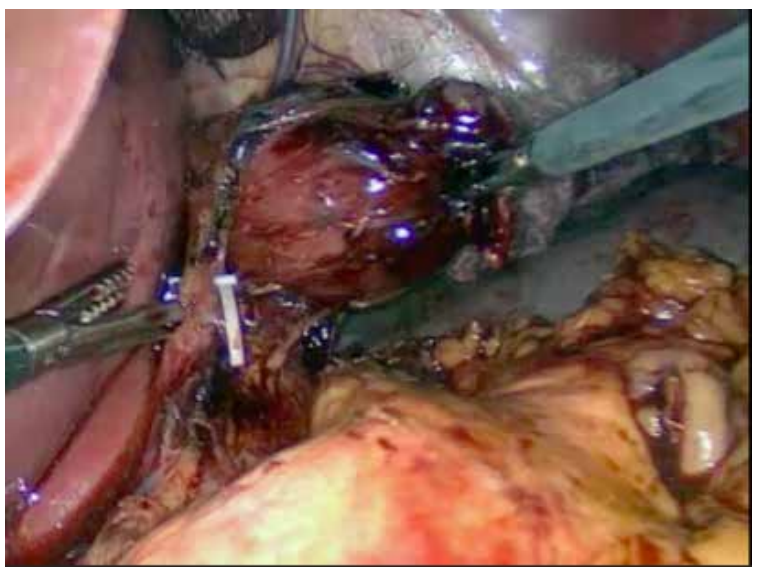

Figura 2. Remanente gástrico del $5 \%$ con preservación de la rama ascendente de la arteria gástrica izquierda. 
cando diferencia de medias (t de student) para variables cuantitativas que distribuyan normal y U de Mann Whitney en caso contrario. Para las variables cualitativas se aplica el $\mathrm{Chi}^{2}$ o test de Fisher. Se establece como significativo un valor de $\mathrm{p} \leq$ 0,05. Se utilizó el Software: SPSS v22 ${ }^{\circledR}$ licencia Universidad CES.

\section{Resultados}

Entre enero de 2017 y junio de 2019 se realizaron 73 gastrectomías casi totales radicales por cáncer gástrico. De ellas, en cinco pacientes la técnica se convirtió a cirugía abierta, seis pacientes tenían datos incompletos en la historia clínica y un paciente requirió esofagectomía con ascenso gástrico, quedando un total de 6I pacientes que cumplieron criterios de inclusión.

El grupo estuvo conformado por 3I hombres y 30 mujeres, el promedio de edad fue de 60,15 años; la mayoría de los pacientes (68\%) presentaban un índice de masa corporal (IMC) normal, y el 8,2 \% algún grado de obesidad. Las comorbilidades encontradas más frecuentemente fueron la hipertensión arterial, el tabaquismo y la diabetes (tabla I).

Dentro de las variables intraoperatorias se encontró la preservación de la RAAGI en el 73,8 \% de los pacientes $(n=45)$. Para la anastomosis gastro-yeyunal se utilizó sutura mecánica con cierre de enterotomía en el 52,5\% de los pacientes $(n=32)$ y sutura manual en dos planos en el $47,5 \%$ de los pacientes $(n=29)$. Ocho pacientes recibieron transfusiones de hemoderivados y ningún paciente requirió uso intraoperatorio de vasopresores.

El promedio de ganglios resecados fue de 24, la disección D2 se logró en el $88 \%$ de los casos $(n=54)$, y en siete de las intervenciones se reportaron menos de $\mathrm{I} 5$ ganglios resecados. En cuatro pacientes la patología informó alguno de los bordes positivos (distal, proximal o radial) y se encontraron cuatro pacientes con enfermedad metastásica, tres de ellos por lavado peritoneal positivo y un paciente con metástasis hepática única, resecada en el mismo acto quirúrgico. Se presentaron un total de Io complicaciones;
Tabla 1. Característica de los pacientes, variables intraoperatorias y variables de patología.

\begin{tabular}{|c|c|c|}
\hline \multicolumn{3}{|l|}{ Características de los pacientes } \\
\hline Variable & $\mathbf{n}$ & $\%$ \\
\hline Sexo (hombre) & 31 & 50,8 \\
\hline \multicolumn{3}{|l|}{ IMC } \\
\hline Bajo peso & 3 & 4,9 \\
\hline Normal & 42 & 68,8 \\
\hline Sobrepeso & 11 & 18,0 \\
\hline Obesidad grado I & 4 & 6,5 \\
\hline Obesidad grado II & 0 & 0 \\
\hline Obesidad grado III & 1 & 1,6 \\
\hline Obesidad grado IV & 0 & 0 \\
\hline \multicolumn{3}{|l|}{ Comorbilidades } \\
\hline HTA & 22 & 36,1 \\
\hline $\mathrm{DM}$ & 10 & 16,4 \\
\hline EAOC & 1 & 1,6 \\
\hline EPOC & 2 & 3,3 \\
\hline Enfermedad Coronaria & 1 & 1,6 \\
\hline Tabaquismo & 22 & 36,1 \\
\hline \multicolumn{3}{|l|}{ Variables intraoperatorias } \\
\hline \multicolumn{3}{|l|}{ Tipo de anastomosis } \\
\hline Manual & 29 & 47,5 \\
\hline Mecánica & 32 & 52,5 \\
\hline Preservación rama ascendente & 45 & 73,8 \\
\hline Transfusiones & 8 & 13,1 \\
\hline \multicolumn{3}{|l|}{ Variables de patología } \\
\hline \multicolumn{3}{|l|}{ Tumor* } \\
\hline 1 & 6 & 9,8 \\
\hline 2 & 16 & 26,2 \\
\hline 3 & 23 & 37,7 \\
\hline 4 & 16 & 26,2 \\
\hline \multicolumn{3}{|l|}{ Nódulos* } \\
\hline 0 & 23 & 37,7 \\
\hline 1 & 17 & 27,9 \\
\hline 2 & 9 & 14,7 \\
\hline 3 & 12 & 19,7 \\
\hline Metástasis* & 4 & 6,5 \\
\hline
\end{tabular}

Según clasificación TNM de AJCC 2017.

HTA: Hipertensión arterial; DM: Diabetes Mellitus; EAOC: Enfermedad arterial oclusiva crónica; EPOC: Enfermedad pulmonar obstructiva crónica.

Fuente: Expediente clínico. Fundación Cancerológica de Colombia - Clínica Vida. 
entre las complicaciones de la anastomosis se encontraron tres estenosis, cuatro filtraciones y un paciente con hemorragia del tubo digestivo superior. Dos pacientes presentaron colecciones intrabdominales (tabla 2).

Se realizó un análisis bivariado comparando tanto las características del paciente como las variables intraoperatorias, con el desenlace compuesto por cualquier tipo de complicación. No se encontraron diferencias estadísticamente significativas para las variables estudiadas, sin embargo, en las variables relacionadas con el reporte de patología, la presencia de enfermedad en ganglios linfáticos en estadios Ni y N2 estuvo asociada con cualquier tipo de complicaciones con diferencia estadísticamente significativa $(\mathrm{p}=0,032)$ (tabla 3$)$. No se encontró asociación de la preservación de la RAAGI con un mayor número de complicaciones en general $(p=0,713)$.

Cuando se analiza específicamente la estenosis de la anastomosis gastro-yeyunal, no se encontraron diferencias estadísticamente significativas al comparar las características de los pacientes (tabla 4). En el $67 \%$ de los pacientes que no presentaron estenosis se realizó técnica con preservación de la rama ascendente, comparado con un $33 \%$ de quienes si presentaron esta complicación $(\mathrm{p}=\mathrm{o}, \mathrm{I} 66)$. La estenosis de la anastomosis se presentó en tres casos, dos con sección de RAAGI y uno con preservación de esta rama, esta diferencia no resultó significativa $(p=0, I 66)$. Ninguna característica asociada al

Tabla 2. Complicaciones en los pacientes sometidos a gastrectomía casi total

\begin{tabular}{lcc}
\hline Complicaciones & $\mathbf{n}$ & \% \\
\hline Filtración & 4 & 6,5 \\
Estenosis & 3 & 4,9 \\
Hemorragia & 1 & 1,6 \\
Colecciones & 2 & 3,3 \\
Totales & 10 & 16,4 \\
\hline
\end{tabular}

Fuente: Expediente clínico. Fundación Cancerológica de Colombia - Clínica Vida.
Tabla 3. Análisis bivariado de las complicaciones totales.

\begin{tabular}{lccc}
\hline \multicolumn{4}{c}{ Complicaciones totales } \\
\hline Variable & SI & NO & p \\
\hline Edad promedio ( \pm DS) & $62,2( \pm 14,74)$ & $59,74( \pm 12,69)$ & 0,588 \\
Ganglios mediana (RIQ) & $24,5(18-34,3)$ & $21(17-30)$ & 0,598 \\
Ganglios positivos & $3,5(0,1-4,5)$ & $1(0-3)$ & 0,064 \\
\hline Sexo (hombre) $n(\%)$ & $5(50)$ & $5(50)$ & 1 \\
\hline IMC $n(\%)$ & & & 0,521 \\
\hline Bajo peso/normal & $6(60)$ & $39(76,5)$ \\
Sobrepeso & $3(30)$ & $8(15,7)$ \\
Obesidad & $1(10)$ & $4(7,8)$ & \\
\hline Comorbilidades $n(\%)$ & & & \\
\hline HTA & $6(60)$ & $16(31,4)$ & 0,147 \\
DM & $3(30)$ & $7(13,7)$ & 0,345 \\
Tabaquismo & $5(50)$ & $17(33,3)$ & 0,473 \\
\hline
\end{tabular}

\begin{tabular}{|c|c|c|c|}
\hline \multicolumn{4}{|l|}{ Variables intraoperatorias } \\
\hline $\begin{array}{l}\text { Tipo de anastomosis } n \\
(\%)\end{array}$ & & & 1 \\
\hline Manual & $5(50)$ & $24(47,1)$ & \\
\hline Mecánica & $5(50)$ & $27(52,9)$ & \\
\hline $\begin{array}{l}\text { Preservación rama as- } \\
\text { cendente }\end{array}$ & & & 0,713 \\
\hline $\mathrm{Si}$ & $7(70)$ & $38(74,5)$ & \\
\hline No & $3(30)$ & $13(25,5)$ & \\
\hline Transfusiones & $2(20)$ & $6(11,8)$ & 0,607 \\
\hline \multicolumn{4}{|l|}{ Variables de patología } \\
\hline Tumor* & & & 0,627 \\
\hline 1 & $1(10)$ & $5(9,8)$ & \\
\hline 2 & $1(10)$ & $15(29,4)$ & \\
\hline 3 & $5(50)$ & $18(35,3)$ & \\
\hline 4 & $3(30)$ & $13(25,5)$ & \\
\hline Nódulos* & & & 0,032 \\
\hline 0 & $1(10)$ & $22(43,1)$ & \\
\hline 1 & $4(40)$ & $13(25,5)$ & \\
\hline 2 & $4(40)$ & $5(9,8)$ & \\
\hline 3 & $1(10)$ & $11(21,6)$ & \\
\hline Metástasis* & $1(10)$ & $3(5,9)$ & 0,521 \\
\hline
\end{tabular}

*Según clasificación TNM de AJCC 2017.

DS: desviación estándar; RIQ: rango intercuartílico; IMC: índice de masa corporal; HTA: hipertensión arterial; DM: diabetes mellitus.

Fuente: Expediente clínico. Fundación Cancerológica de Colombia Clínica Vida. 
Tabla 4. Análisis bivariado en pacientes con estenosis de la anastomosis.

\begin{tabular}{|c|c|c|c|}
\hline \multirow[b]{2}{*}{ Variable } & \multicolumn{2}{|c|}{ Estenosis } & \multirow[b]{2}{*}{$p$} \\
\hline & Si & No & \\
\hline Edad mediana (RIQ) & $68(59-73)$ & $60(53,8-69)$ & 0,535 \\
\hline Ganglios mediana (RIQ) & $21(16-23)$ & $22,5(17-31)$ & 0,493 \\
\hline $\begin{array}{l}\text { Ganglios positivos } \\
\text { mediana (RIQ) }\end{array}$ & $4(2,5-5)$ & $1(0-4)$ & 0,239 \\
\hline Sexo (hombre) n (\%) & $1(33,3)$ & $30(51,7)$ & 0,612 \\
\hline IMC n (\%) & & & 0,571 \\
\hline Bajo peso/normal & $3(100)$ & $42(72,4)$ & \\
\hline Sobrepeso & 0 & $11(19)$ & \\
\hline Obesidad & 0 & $5(8,6)$ & \\
\hline \multicolumn{4}{|l|}{ Comorbilidades n (\%) } \\
\hline HTA & $1(33,3)$ & $21(36,2)$ & 1 \\
\hline DM & $1(33,3)$ & $9(15,5)$ & 0,421 \\
\hline Tabaquismo & $1(33,3)$ & $21(36,2)$ & 1 \\
\hline \multicolumn{4}{|l|}{ Variables intraoperatorias } \\
\hline Tipo de anastomosis n (\%) & & & 0,600 \\
\hline Manual & $2(66,7)$ & $27(46,6)$ & \\
\hline Mecánica & $1(33,3)$ & $31(53,4)$ & \\
\hline $\begin{array}{l}\text { Preservación rama } \\
\text { ascendente } \mathrm{n}(\%)\end{array}$ & & & 0,166 \\
\hline $\mathrm{Si}$ & $1(33,3)$ & $44(75,9)$ & \\
\hline No & $2(66,7)$ & $14(24,1)$ & \\
\hline Transfusiones n (\%) & $1(33,3)$ & $7(12,1)$ & 0,349 \\
\hline \multicolumn{4}{|l|}{ Variables de patología } \\
\hline Tumor n (\%) & & & 0,444 \\
\hline 1 & $1(33,3)$ & $5(8,6)$ & \\
\hline 2 & 0 & $16(27,6)$ & \\
\hline 3 & $1(33,3)$ & $22(37,9)$ & \\
\hline 4 & $1(33,3)$ & $15(25,9)$ & \\
\hline Nódulos* n (\%) & & & 0,235 \\
\hline 0 & 0 & $23(39,7)$ & \\
\hline 1 & $2(66,7)$ & $15(25,9)$ & \\
\hline 2 & $1(33,3)$ & $8(13,8)$ & \\
\hline 3 & 0 & $12(20,7)$ & \\
\hline Metástasis* & $1(33,3)$ & $3(5,2)$ & 0,187 \\
\hline
\end{tabular}

*Según clasificación TNM de AJCC 2017.

RIQ: rango intercuartílico; IMC: índice de masa corporal; HTA: hipertensión arterial; DM: diabetes mellitus

Fuente: Expediente clínico. Fundación Cancerológica de Colombia - Clínica Vida.
Tabla 5. Análisis bivariado en pacientes con filtración de la sutura.

\begin{tabular}{|c|c|c|c|}
\hline \multicolumn{4}{|c|}{ Filtración } \\
\hline Variable & SI & NO & $p$ \\
\hline Edad promedio ( $\pm \mathrm{DS})$ & $63(45,5-75,5)$ & $60(54,0-69)$ & 0,811 \\
\hline Ganglios mediana (RIQ) & $25(19,5-32)$ & $21(17-30)$ & 0,603 \\
\hline $\begin{array}{l}\text { Ganglios positivos } \\
\text { mediana (R|Q) }\end{array}$ & $2(0,5-3,5)$ & $1(0-4)$ & 0,709 \\
\hline Sexo (hombre) n (\%) & $3(75)$ & $28(49,1)$ & 0,612 \\
\hline IMC n (\%) & & & 0,383 \\
\hline Bajo peso/normal & $2(50)$ & $43(75,4)$ & \\
\hline Sobrepeso & $1(25)$ & $10(17,5)$ & \\
\hline Obesidad & $1(25)$ & $4(7)$ & \\
\hline \multicolumn{4}{|l|}{ Comorbilidades n (\%) } \\
\hline HTA & $2(50)$ & $20(35,1)$ & 0,615 \\
\hline DM & 0 & $10(17,5)$ & 1 \\
\hline Tabaquismo & $2(50)$ & $20(35,1)$ & 0,615 \\
\hline \multicolumn{4}{|l|}{ Variables intraoperatorias } \\
\hline \multicolumn{4}{|l|}{ Tipo de anastomosis $\mathrm{n}(\%)$} \\
\hline Manual & $2(50)$ & $27(47,4)$ & 1 \\
\hline Mecánica & $2(50)$ & $30(52,6)$ & \\
\hline $\begin{array}{l}\text { Preservación rama ascen- } \\
\text { dente } n(\%)\end{array}$ & & & 1 \\
\hline $\mathrm{Si}$ & $3(75)$ & $42(73,7)$ & \\
\hline No & $1(25)$ & $15(26,3)$ & \\
\hline Transfusiones & 0 & $8(14)$ & 1 \\
\hline \multicolumn{4}{|l|}{ Variables de patología } \\
\hline Tumor* & & & 0,676 \\
\hline 1 & 0 & $6(10,5)$ & \\
\hline 2 & $1(25)$ & $15(26,3)$ & \\
\hline 3 & $1(25)$ & $22(38,6)$ & \\
\hline 4 & $2(50)$ & $14(24,6)$ & \\
\hline Nódulos* & & & 0,556 \\
\hline 0 & $1(25)$ & $22(38,6)$ & \\
\hline 1 & $2(50)$ & $15(26,3)$ & \\
\hline 2 & $1(25)$ & $8(14)$ & \\
\hline 3 & 0 & $12(21,1)$ & \\
\hline Metástasis* & 0 & $4(7)$ & 1 \\
\hline
\end{tabular}

*Según clasificación TNM de AJCC 2017

DS: desviación estándar; RIQ: rango intercuartílico; IMC: índice de masa corporal; HTA: Hipertensión arterial. DM: Diabetes Mellitus

Fuente: Expediente clínico. Fundación Cancerológica de Colombia Clínica Vida. 
TNM presentó diferencias cuando se comparó la presencia o no de estenosis en la anastomosis gastro-yeyunal.

Cuando se compararon los pacientes con filtración de la anastomosis gastro-yeyunal con quienes no la presentaron, no se encontraron diferencias entre los grupos según las características del paciente, variables intraoperatorias y características asociadas al resultado de la patología (tabla 5). En el posoperatorio, solo un paciente tuvo hemorragia del tubo digestivo superior asociada a la anastomosis, por lo cual no se realizó comparación para este desenlace.

Por no encontrar relación entre la preservación de RAAGI y las complicaciones de la anastomosis, y otras variables de confusión, no se realizó análisis multivariado.

\section{Discusión}

En pacientes con cáncer gástrico, la cirugía de resección gástrica laparoscópica se posiciona actualmente como un procedimiento seguro y que probablemente aumentará en frecuencia a futuro. El perfeccionamiento de esta técnica podría llevar a una menor presentación de complicaciones intra y postoperatorias. Parte del éxito de esta técnica quirúrgica reside en poder conservar la RAAGI, realizando un vaciamiento ganglionar adecuado, siempre y cuando la neoplasia gástrica no involucre directamente esta rama o se sacrifiquen los términos oncológicos de esta cirugía.

La importancia de la preservación de las ramas de la arteria gástrica izquierda en cirugía oncológica no ha sido estudiada hasta el momento. Sin embargo, en cirugía bariátrica se ha descrito su importancia en un estudio con cadáveres en donde se encontró que, la realización de una manga gástrica podría causar una debilidad en el remanente gástrico debido al engrapado de ramas de la arteria gástrica izquierda, y que dicha debilidad podría exacerbar disparidades en la demanda vascular, causando la aparición de futuras complicaciones ${ }^{8}$.

En la literatura, la técnica quirúrgica con preservación de la RAAGI junto con las ramas celiaca y hepática del nervio vago, se ha descrito en cirugía abierta. Se realizó en cinco pacientes, observando posteriormente un funcionamiento normal de la vesícula biliar confirmado por medio de ecografía, no se encontró regurgitación de bario en el estudio contrastado y no hubo aparición de síntomas digestivos en el postquirúrgico ${ }^{9}$. Este estudio favorece la hipótesis de que el paciente con preservación de la RAAGI también conserva ramas del nervio vago, lo que mejoraría la calidad de vida en cuanto a la ausencia de síntomas digestivos en el postquirúrgico.

Azagra, et al., describió la técnica de gastrectomía radical casi total con remanente gástrico del $5 \%$ por laparoscopia ${ }^{\mathrm{IO}}$, donde mencionan que la irrigación del remanente gástrico proviene de las ramas esofágicas de la arteria gástrica izquierda, de la arteria diafragmática inferior izquierda o de las arterias frénica y gástrica. Posteriormente se han publicado resultados a corto plazo de esta técnica laparoscópica, con 67 pacientes operados con intención curativa, Io por cáncer gástrico precoz y 57 avanzados, logrando resultados excelentes, sin conversión a cirugía abierta, sin presentar casos de fugas de la anastomosis gastro-yeyunal y observando solo un caso de hemorragia del tubo digestivo superior ${ }^{\mathrm{II}}$.

El estudio $K L A S S^{12}$ que compara resultados a corto plazo entre cirugía abierta y laparoscópica para gastrectomía distal, reporta para cirugía laparoscópica una morbilidad del I6,6 \%, resultado muy similar a las complicaciones de nuestro estudio que fueron del $16,39 \%$, sin embargo, la conversión a cirugía abierta en nuestro caso fue de $8,20 \%$, el doble de lo reportada por la literatura $(4,13 \%)$.

Al comparar específicamente las complicaciones relacionadas con la anastomosis, el estudio KLASS no reporta casos de estenosis y solo un I,8\% de filtraciones ${ }^{12}$. Nuestra serie encontró un número mayor de complicaciones relacionadas con la anastomosis gastro-yeyunal $(4,9 \mathrm{I} \%$ de estenosis y $6,55 \%$ de filtración), lo que puede deberse a la dificultad de reproducir la experiencia reportada por los países orientales, donde la incidencia por cáncer gástrico es muy alta, lo que 
ha llevado al desarrollo de centros de excelencia, con amplia experiencia en el tratamiento de esta patología.

Kelly, et al, mostraron su experiencia con la gastrectomía laparoscópica en los Estados Unidos ${ }^{13}$, observando un $20 \%$ de complicaciones totales. En cuanto a las complicaciones relacionadas con la anastomosis, encontraron que cuatro pacientes $(4,60 \%)$ presentaron filtración y tres pacientes $(3,45 \%)$ estenosis.

En Colombia, se presentó la experiencia del Instituto Nacional de Cancerología, en Bogotá, en el manejo quirúrgico laparoscópico del cáncer del tracto digestivo superior ${ }^{14}$, informando excelentes resultados en 22 pacientes a quienes se les realizo gastrectomía laparoscópica; solamente un paciente presentó filtración y en otro ocurrió estenosis de la anastomosis, datos muy similares a los presentados en nuestra serie.

En el análisis bivariado, no se logró identificar alguna relación significativa entre las variables de los pacientes y las variables intraoperatorias, con cualquier complicación de la anastomosis. Sin embargo, clínicamente parece ser que la no conservación de la RAAGI en pacientes con remanente gástrico pequeño puede asociarse a una mayor frecuencia de estenosis de la anastomosis $(66,7 \%$ versus $33,3 \%)$, sin que esto tenga una significancia estadística $(\mathrm{p}=0, \mathrm{I66})$.

En comparación con los estudios previamente descritos, las complicaciones de la anastomosis son un poco más frecuente en nuestra cohorte. No parece existir una relación entre las complicaciones de la anastomosis y la preservación de la RAAGI, por lo que es necesario continuar la investigación en búsqueda de otros factores asociados.

En esta serie, debido a la muestra pequeña y la escasa frecuencia de complicaciones de la anastomosis, es difícil encontrar asociaciones estadísticamente significativas. La única variable que muestra estar relacionada con las complicaciones en general, es la enfermedad ganglionar en estadios Ni y N2, esto puede estar relacionado con el hecho de tratarse de una enfermedad más avanzada.
Dentro de las limitaciones del estudio hay que tener en cuenta que muchas de estas complicaciones pueden relacionarse con el estado nutricional de los pacientes, el cual resulta muy difícil de valorar en la historia clínica por falta de datos y por reportes de laboratorio incompletos, además la mayoría de pacientes en esta cohorte corresponden al estado locoregional de cáncer gástrico y muy pocos a enfermedad localizada. El carácter retrospectivo del estudio tiene la limitante de la calidad de información de la fuente primaria.

Por otro lado, el estudio tiene la fortaleza de mostrar los resultados de un centro con experiencia en laparoscopia avanzada y demuestra la factibilidad de la técnica de la gastrectomía casi total por laparoscopia. La información fue estandarizada en el instrumento de recolección y recolectada por cirujanos con experiencia en el tema.

\section{Conclusiones}

Este estudio describe la morbilidad asociada a la técnica de gastrectomía laparoscópica con preservación de la RAAGI en una cohorte de pacientes. Se recomienda estudiar esta relación y la calidad de vida de los pacientes con remanentes gástricos pequeños, en grupos con una mayor muestra de pacientes.

La gastrectomía casi total con remanente gástrico del $5 \%$ y preservación de la RAAGI por laparoscopia es una técnica factible y cumple con los requisitos oncológicos de resección radical en pacientes con cáncer gástrico. La variante de la técnica tradicional no se relaciona con aumento en las complicaciones de la anastomosis gastro-yeyunal.

\section{Cumplimiento de normas éticas}

Consentimiento informado: Se obtuvo aprobación del protocolo de investigación por parte del comité de ética de la universidad CES (acta No 223 del 23 de septiembre de 2019) y del comité de ética de la Fundación Cancerológica de Colombia - Clínica Vida (acta No 67). 
Declaración de conflictos de interés: los autores declaran que no tienen conflictos de interés. Fuentes de financiación: este trabajo fue financiado por los autores.

\section{Referencias}

I. National Comprehensive Cancer Network (NCCN) Clinical Practice Guidelines in Oncology. Fecha de consulta: Io de noviembre de 2019. Disponible en: https://www.nccn.org/professionals/physician_gls/default.aspx\#site

2. Dumont F, Thibaudeau E. Gastrectomía por cáncer. EMC - Técnicas Quirúrgicas - Aparato Digestivo. 20I8;34:I-23. https://doi.org/IO.IOI6/SI282-9I29(I8)899I2-3

3. Losanoff JE, Richman BW, Jones JW. Distal subtotal gastrectomy with preservation of the left gastric artery and vagal branches. Am J Surg. 2003;185:395-6. https://doi.org/IO.IOI6/Sooo2-96Io(O2)OI426-5

4. Huang Y, Mu G-C, Qin X-G, Chen Z-B, Lin J-L, Zeng $\mathrm{Y}-\mathrm{J}$. Study of celiac artery variations and related surgical techniques in gastric cancer. World J Gastroenterol. 2015;2I:6944-5I. https://doi.org/I0.3748/wjg.v2I.i22.6944

5. Yuasa Y, Okitsu H, Goto M, Kuramoto S, Tomibayashi A, Matsumoto D, et al. Three-dimensional CT for preoperative detection of the left gastric artery and left gastric vein in laparoscopy-assisted distal gastrectomy: Gastric vessel detected by 3-D CT in LADG. Asian J Endosc Surg. 20I6;9:I79-85. https://doi.org/IO.IIII/ases.I2280

6. Takagi H, Morimoto T. Near-total gastrectomy. J Surg Oncol. 1984;26:I4-6. https://doi.org/IO.IOO2/jso.2930260105

7. Salo JA, Saario I, Kivilaakso EO, Lempinen M. Near-Total gastrectomy for gastric cancer. Am J Surg. I988;I55:486-9.

https://doi.org/Io.IoI6/Sooo2-96Io(88)80II9-3
8. Perez M, Brunaud L, Kedaifa S, Guillotin C, Gerardin A, Quilliot D, et al. Does Anatomy Explain the Origin of a Leak after Sleeve Gastrectomy? Obes Surg. 20I4;24:I71723. https://doi.org/IO.IO07/sII695-OI4-I256-4

9. Hagiwara A, Imanishi T, Sakakura C, Otsuji E, Kitamura K, Itoi $\mathrm{H}$, et al. Subtotal gastrectomy for cancer located in the greater curvature of the middle stomach with prevention of the left gastric artery. Am J Surg. 2002;183:692-6. https://doi.org/Io.IoI6/Sooo2-96Io(02)00854-I

Io. Arru L, Azagra JS, Facy O, Makkai-Popa ST, Poulain V, Goergen M. Totally laparoscopic $95 \%$ gastrectomy for cancer: technical considerations. Langenbecks Arch Surg. 2015;400:387-93. https://doi.org/I0.I007/s00423-0I5-I283-I

II. Sarriugarte A, Arru L, Makai-Popa S, Goergen M, Ibañez-Aguirre FJ, Azagra JS. Resultados a corto plazo de la gastrectomía casi total (95\%gastrectomy) laparoscópica. Cir Esp. 2018;96:634-9. https://doi.org/I0.I0I6/j.ciresp.20I8.06.009

I2. Lee H-J, Hyung WJ, Yang H-K, Han SU, Park Y-K, An JY, et al. Short-term Outcomes of a Multicenter Randomized Controlled Trial Comparing Laparoscopic Distal Gastrectomy With D2 Lymphadenectomy to Open Distal Gastrectomy for Locally Advanced Gastric Cancer (KLASS-02-RCT). Ann Surg. 2019;27:983-9I.

https://doi.org/I0.I097/SLA.00ooooooooo03217

I3. Kelly KJ, Selby L, Chou JF, Dukleska K, Capanu M, Coit DG, et al. Laparoscopic Versus Open Gastrectomy for Gastric Adenocarcinoma in the West: A Case-Control Study. Ann Surg Oncol. 2015;22:3590-6. https://doi.org/IO.I245/sIO434-0I5-438I-y

I4. Borráez Segura BA, Oliveros R, Sánchez R, Cajiao JC, Pinilla RE. Primera experiencia de cirugía laparoscópica en cáncer del tracto digestivo superior. Rev Gastroenterol Peru. 20I8;38:340-4. 